\title{
Battery-free Visible Light Sensing
}

\author{
Ambuj Varshney \\ Uppsala University, Sweden \\ ambuj.varshney@it.uu.se \\ Luca Mottola \\ Politecnico di Milano, Italy \\ RISE SICS, Sweden \\ luca@sics.se
}

\author{
Andreas Soleiman \\ Uppsala University, Sweden \\ andreas.soleiman@it.uu.se \\ Thiemo Voigt \\ Uppsala University, Sweden \\ RISE SICS, Sweden \\ thiemo@sics.se
}

\begin{abstract}
We present the design of the first Visible Light Sensing (VLS) system that consumes only tens of $\mu \mathrm{Ws}$ of power to sense and communicate. Unlike most existing VLS systems, we require no modification to the existing light infrastructure since we use unmodulated light as a sensing medium. We achieve this by designing a novel mechanism that uses solar cells to achieve a sub- $\mu \mathrm{W}$ power consumption for sensing. Further, we devise an ultra-low power transmission mechanism that backscatters sensor readings and avoids the processing and computational overhead of existing sensor systems. Our initial results show the ability to detect and transmit hand gestures or presence of people up to distances of $330 \mathrm{~m}$, at a peak power of $20 \mu \mathrm{Ws}$. Further, we demonstrate that our system can operate in diverse light conditions ( $100 \mathrm{~lx}$ to $80 \mathrm{klx}$ ) where existing VLS designs fail due to saturation of the transimpedance amplifier (TIA).
\end{abstract}

\section{KEYWORDS}

Visible Light Sensing, Battery-free sensing, Backscatter

\section{INTRODUCTION}

Visible light is a ubiquitous medium that provides illumination to spaces or objects through low-cost fluorescent bulbs, light emitting diodes (LEDs) or natural light. Further, visible light can be sensed using simple and low-cost photodiodes or solar cells which requires minimal processing effort at the sensing device. Thus, visible light offers a significant advantage for sensing applications over mediums such as radio frequency (RF) signals which require complex processing and radios operating in licensed spectrum. However, despite clear advantages, there have only been limited deployments (excluding vision systems) of visible light sensing (VLS) systems.

One of the reasons for the lack of pervasive deployment of VLS systems is that a majority of existing VLS systems fail to take advantage of the ubiquitous nature of visible light, since they use visible light communication (VLC). VLC requires retrofitting of the luminaries with specialized driving circuits [6-8] to modulate

Permission to make digital or hard copies of all or part of this work for personal or classroom use is granted without fee provided that copies are not made or distributed for profit or commercial advantage and that copies bear this notice and the full citation on the first page. Copyrights for components of this work owned by others than ACM must be honored. Abstracting with credit is permitted. To copy otherwise, or republish, to post on servers or to redistribute to lists, requires prior specific permission and/or a fee. Request permissions from permissions@acm.org.

VLCS'17, October 16, 2017, Snowbird, UT, USA

(C) 2017 Association for Computing Machinery.

ACM ISBN 978-1-4503-5142-3/17/10 . \$ \$15.00

https://doi.org/10.1145/3129881.3129890

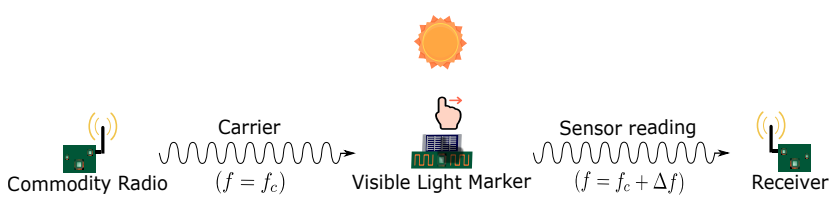

Figure 1: Shadow sensing at $\mu \mathrm{W} s$ of power. We can detect and communicate shadow events by reflecting ambient RF signals at a total power consumption of $20 \mu \mathrm{Ws}$.

visible light, e.g., with beacon information. Modifying existing lighting infrastructure significantly increases the cost and complexity of deployment [14]. Further, existing VLC systems use complex reception logic and circuitry to perform demodulation [6], or FFT operations $[7,8]$ which further hinders pervasive deployment of VLS systems. Thus, a key enabler to achieve widespread deployment of VLS systems is to use unmodulated ambient light for sensing.

Ambient light as a sensing medium allows tracking of shadows cast by objects or people. Recent efforts show that sensing changes in the cast shadow enables human sensing capability [7, 8], or tracking of hand gestures [3]. However, several limitations remain that hinder widespread deployment: First, some prior shadow sensing systems use modulated light $[7,8]$ which, as discussed above, makes the deployment complex. Second, these systems [3, 7, 8] employ conventional light sensing mechanisms.

Conventional light sensing mechanisms [3, 7, 8] employ sensors with components that negatively affect pervasive deployment: they amplify the signals from photodiodes using transimpedance amplifiers (TIA), sample using analog to digital converters (ADCs), and process using computational blocks involving microcontrollers (MCUs) or field-programmable gate arrays (FPGAs). Further, the events are communicated using traditional RF radios [1] or external cables $[7,8]$. However, there are three main problems with this approach: First, it consumes significant amounts of power (mWs) and hence requires sensors that are battery-powered or powered through other external sources. Second, TIAs suffer from saturation under bright light conditions [3] which prevents continuous operation under natural and ambient light. Finally, all of these components significantly increase the cost of sensors, which makes it challenging and expensive to deploy at scale.

Contributions. Simple and ultra-low cost and power light sensors with the ability to sense changes in ambient light can make VLS systems pervasive. Such sensors could operate on small amounts of energy harvested from ambient light or other harvestable sources, and transform any well-lit surface to a sensing medium. The low cost could enable deployment of such sensors at a wide scale. 
We introduce the first VLS system that can sense shadow events and communicate them at tens of $\mu \mathrm{Ws}$ of power. Our system achieves this through ultra-low power and inexpensive sensors which we design. We call these sensors, shown in Figure 2, visible light markers (VLMs). In designing VLMs, we make the following contributions:

- To overcome the high power consumption for sensing, our contribution is to use solar cells. Similar to photodiodes, solar cells exhibit a change in voltage when the light conditions change. In comparison to photodiodes which require energy-expensive TIAs, solar cells are passive and can harvest energy. Further, unlike TIAs, solar cells can operate under diverse light conditions (extremely bright to very low light). However, the use of solar cells in VLS systems is unexplored.

- A key challenge when operating battery-free is to consume $\mu \mathrm{Ws}$ of power to perform processing. Existing designs consume significant energy for processing when digitising and communicating shadow events. We achieve sub- $\mu \mathrm{W}$ power consumption by using a thresholding circuit to digitise changes in the ambient light. We introduce the first design that couples solar cells with a thresholding circuit and consumes sub- $\mu \mathrm{Ws}$ for sensing.

- To overcome the overhead from communication of events, we use RF backscatter. We embrace Zhang et al.'s observation [15] that processing is significantly more expensive than backscatter transmissions. We devise a mechanism called Scatterlight that eliminates local processing, and replicates digital signals (thresholding circuit) onto backscatter signals without involving energyexpensive computational blocks.

Combining the three contributions results in the system illustrated in Figure 1. It consists of a carrier signal source, the VLM, and a device to receive and process the backscattered signals.

Our preliminary results demonstrate that our system achieves unprecedented energy efficiency for detection and communication of shadow sensing events. The novel design of a solar cell with thresholding circuit enables us to sense events at a power consumption of $0.5 \mu \mathrm{Ws}$. When coupled with Scatterlight, we can communicate events at a peak power consumption of $20 \mu \mathrm{Ws}$. The power consumption is three orders of magnitude lower when compared to designs used by state-of-the-art systems $[3,7,8]$. Our initial results demonstrate the ability to sense and communicate gestures at significantly lower power consumption when compared to existing designs. Further, we can operate in very bright light conditions ( $>$ $80 \mathrm{klx}$ ), where existing VLS designs suffer from saturation.

\section{RELATED WORK}

Our work is related to the following:

Shadow sensing. Prior work has explored shadow sensing. Li et al.'s system uses an array of photodiodes deployed on the floor together with modulated lights to track user gestures [7, 8]. Kaholokula et al. detect hand gestures by tracking changes in light levels caused by hand motions above multiple photodiodes under unmodulated ambient light [3]. However, all of the above systems used conventional light sensing mechanisms which involves photodiodes coupled with TIAs, and ADCs for sampling on COTS platforms (Arduinos). These mechanisms are energy expensive ( $\mathrm{mW})$, and are susceptible to saturation under bright light.

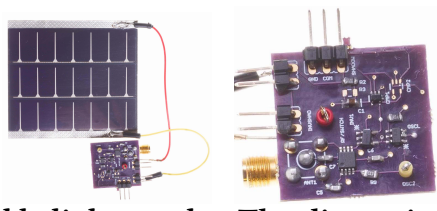

Figure 2: Visible light marker The dimensions of VLM and solarcell are $25 \times 25 \mathrm{~mm}$ and $75 \times 60 \mathrm{~mm}$ respectively

Solar cells as light sensors. Solar cells as light sensors have been restricted to high-speed VLC systems. For example, Wang et al. use a solar panel as a light sensor coupled to an energy-expensive voltage amplifier and ADCs, and receive using a complex OFDM modulation scheme [13]. However, these designs are not suited for battery-free systems due to their high power consumption.

Unmodulated ambient light for sensing. Zhang et al. [16] and Shu et al.[17] demonstrate that indoor lights exhibit a characteristic frequency which enables indoor localisation applications. These systems are similar to our system in terms of using unmodulated light and require no infrastructure modifications. However, they use smartphones for sensing which consumes mWs of power.

Processing overhead. Prior attempts $[10,15]$ have investigated the processing overhead on backscatter sensors. Zhang et al. optimise the computational block, and demonstrate that processing is more energy expensive than backscatter transmissions. Talla et al. [10], in a concurrently released work, design a battery-free cellphone. Their design is similar to Scatterlight in terms of eliminating computational blocks. However, we make significant improvements over their design: Talla et al. backscatter at the same frequency as the carrier signal and thus encounter severe selfinterference [16]. This requires complex processing on expensive software-defined-radio (SDRs) to receive the backscattered signals. In comparison, Scatterlight without involving computation blocks can also shift the backscatter signal away from the carrier, thereby significantly reducing self-interference, which allows the use of low-cost transceivers for receptions.

\section{SYSTEM DESIGN}

\subsection{Overview}

The fundamental operation of our system is to detect and transmit changes in ambient light in order to identify events such as the presence of people or hand gestures. Towards this end, our system performs a series of steps which we describe next:

We first generate a carrier signal so that the VLM can backscatter shadow events. We describe this step in Section 3.2. Next, as we describe in Section 3.3.1, VLM leverages solar cells to harvest energy for its operation and sense shadow events. The analog changes are digitised by using an ultra-low power thresholding circuit, as we describe in Section 3.3.2. These changes are mirrored onto unique backscattered signals using the Scatterlight mechanism, as we describe in Section 3.3.3. Finally, the backscattered events are received and processed by the end-devices to interpret the type of event, as explained in Section 3.4.

\subsection{Ambient RF signal}

Motivation. To enable ultra-low power consumption we use RF backscatter transmissions in order to communicate shadow events. 


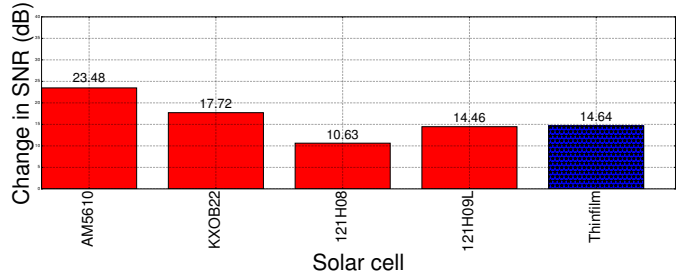

Figure 3: Sensing using a solar cell. A shadow cast on a solar cell causes a significant change in the SNR.

Backscatter works by reflecting ambient RF signals such as unmodulated carriers to communicate $[5,11,16]$ the desired information.

Generating a carrier signal. To generate a carrier signal, we use the test mode available on transceivers [11]. We generate a carrier signal at a frequency of $868 \mathrm{MHz}$ and of strength $24 \mathrm{dBm}$ using a TI CC1310 transceiver together with an external power amplifier. Recent systems also demonstrate the ability to generate carrier signals from BLE [2], WiFi [11] and 802.15.4 radios [11]. Our system can operate unmodified in the $2.4 \mathrm{GHz}$ band, and utilise the vast deployment of these radios to provide a carrier signal.

\subsection{Visible light markers}

The crucial components for the VLM design are: solar cells as light sensors, an ultra-low power digitisation mechanism, and the use of Scatterlight to communicate the shadow events.

\subsubsection{Light sensor.}

Motivation. Existing VLC-based systems commonly employ photodiodes to sense rapid changes in visible light $[7,8]$. The photodiodes are coupled with a TIA to amplify the signal to the level required for further processing. The use of TIAs, however, increases the energy consumption to mWs, making it difficult to operate the sensors on harvested energy. Further, TIAs are also susceptible to saturation under bright light $[3,7,8]$.

Using solar cells as light sensors. We take advantage of solar cells to achieve the necessary amplification without energyexpensive amplifiers. Similar to photodiodes, solar cells transform variations in ambient light caused by, e.g., a shadow to a change in the electrical signal. Moreover, solar cells are passive and do not require an external power source. More importantly, solar cells can operate in diverse light conditions without suffering from saturation $[3,7]$ that affects existing TIA designs (see Section 4). Solar cells have been recently used to enable high-speed VLC [13] which is not applicable for systems that operate on harvested energy, since they make use of energy-hungry ADCs and extensive processing. Selecting a solar cell. To enable our vision of VLM, it is important to select a solar cell that can be used for shadow sensing, harvesting and can be easily deployed. We evaluate six different commercially available solar cells of different characteristics to select the one that is the most responsive when sensing the presence of a shadow. We perform an experiment indoors under a light level of $200 \mathrm{~lx}$, which is representative of illumination levels found indoors. We place a solar cell on the floor, and cast a shadow on it. We track its analog output using a logic analyser. We perform the experiment three times for each solar cell. Figure 5 demonstrates that all six solar cells observe a significant change ( $>10 \mathrm{~dB})$ in the signal-to-noise ratio (SNR) which confirms that a solar cell can be used for passive sensing. We find the best performing solar cell to be Sanyo AM5610, Ixys KXOB22 and the Powerfilm thinfilm Mp3-37. The Thinfilm solar cell offers the highest short-circuit current which improves energy harvesting performance. Further, it has a form factor that allows it to be easily placed on clothes or on the floor. Thus, we select the thin film (USD 4) solar cell for the VLM. We note, however, in the present design we use a single and large solar cell for sensing which limits the spatial resolution. In the future, we will integrate multiple tiny solar cells to the VLM in order to improve this.

\subsubsection{Digitisation.}

Motivation. Existing VLS systems also consume significant energy to process and convert analog signals to the digital domain due to the use of energy expensive ADCs [7, 8]. The overhead is substantial for sensing systems [10] where high frequency ADC sampling operations are required to detect shadows or other sensing events. Digitising at $\mu \mathrm{Ws}$ of power. We employ a thresholding circuit in place of commonly employed ADCs to convert changes in analog signals to binary values, thus acting as a 1-bit ADC. Using a thresholding circuit for sensing brings two key advantages over ADCs: First, a thresholding circuit enables ultra-low power digitisation of analog signals. Second, as opposed to sampling an analog signal using an ADC, a thresholding circuit is always active, and it only digitises when there is a change in the input analog signal. A thresholding circuit, as shown in Figure 4 consists of a comparator and a low pass filter (LPF) composed of a resistor (R) and a capacitor (X1). Crucial to the operation of the thresholding circuit is the selection of $\mathrm{R}$ and $\mathrm{X} 1$ which dictates the slowest change in visible light that can be digitised. We select values for these components to detect phenomenon that occur at the timescale of milliseconds such as values hand gestures or presence detection. Thresholding circuits are commonly employed in backscatter systems [9], and low-power gesture-recognition systems [4]. However, to the best of our knowledge, we are the first to use a thresholding circuit with a solar cell to achieve sub $\mu \mathrm{Ws}$ of power for VLS. In our implementation of the thresholding circuit, we use an ST microelectronics TS881 comparator (USD 0.3).

\subsubsection{Scatterlight.}

Motivation. Processing sensor values is energy expensive due to computational blocks [10, 15]. Existing VLS systems leverage platforms such as an Arduino to preprocess light samples, and transfer these to an end-device such as a workstation [7, 8].

To eliminate the overhead of local processing, we embrace Zhang et al.'s observation [15] that processing is significantly more energy expensive than backscatter transmissions. We present a mechanism we call Scatterlight that can communicate shadow events without using energy-expensive computational blocks such as FPGAs or MCUs that are commonly employed on sensors $[7,8,15]$.

Scatterlight mechanism on VLM. The concept behind Scatterlight is quite simple: We replicate changes in backscattered signals similar to changes encountered in light conditions due to a shadow event. However, we face several challenges in order to realise the concept: First, the carrier adds significant interference to the weak backscatter signals. Second, we have to modulate the carrier signal 


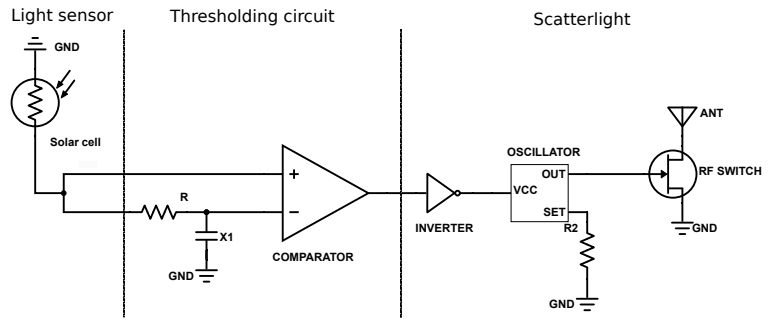

Figure 4: Visible light marker schematic. VLM avoids energy expensive components and local processing. VLM can detect and communicate shadow events at power of $20 \mu \mathrm{W}$.

with shadow events without using computational blocks. Finally, the Scatterlight mechanism should be able to support concurrent transmissions from VLMs at the same time.

We solve the first challenge by building on recent systems $[5,16]$ which keep the backscattered signal and the carrier at separate frequencies: If the ambient $\mathrm{RF}$ signal is present at a center frequency of $f_{c}$, while the VLM backscatters at a frequency of $\Delta f$, the backscattered signal appears at an offset $\Delta f$ away from $f_{c}$. This displacement reduces interference from the carrier $[5,16]$, while allowing the receiver to detect backscattered signals. However, a crucial question is the choice of $\Delta f$, which is transceiver dependent. We employ a transceiver that requires a $\Delta f$ of $100 \mathrm{kHz}$ [11].

To overcome the second challenge, we observe that a commodity radio transceiver enables fast RSSI sampling to determine the energy levels, e.g., due to the presence of backscatter transmissions [16]. If we can backscatter for the duration of the shadow event, the receiver can detect the presence and duration of the shadow events by observing backscatter signals in the RSSI samples.

We implement Scatterlight and achieve the above capability by building on the fact that a digital signal from the thresholding circuit can directly control a backscatter switch, thus modulating the carrier with information of the shadow event. However, the key challenge with such a design is that backscatter signals are at the same frequency as the carrier signal which causes severe selfinterference [10]. Further, supporting multiple VLMs becomes challenging as the tags backscatter at the same frequency. To overcome these challenges, as we illustrate in the Figure 4, we instead control an ultra-low power oscillator (LTC 6906, USD 1.5) through a thresholding circuit. The oscillator controls the backscatter switch (NXP BFT25A, USD 0.2). We configure the oscillator to a frequency larger than $\Delta f$ required to mitigate self-interference. Thus, when there is a shadow event, the thresholding circuit enables the oscillator to generate a backscatter signal at a frequency $\Delta f$ from the carrier $f_{c}$.

Finally, to support concurrent transmissions from VLMs, we devise a scheme borrowed from frequency division multiple access (FDMA). In the scheme, we allocate each VLM a distinct frequency (by configuring the oscillator) which is mapped to an identification number. The receiver, by sampling at these frequencies, can distinguish transmissions from individual VLMs.

\subsection{Reception at end-devices}

We receive Scatterlight transmissions from the VLM at the enddevices. To receive transmissions, we sample the RSSI at the transmit frequency of the VLM. When supporting multiple tags, we sequentially sample the different operational frequencies of tags.
Table 1: Power consumption breakdown of VLM at $2 \mathrm{~V}$

\begin{tabular}{|l|l|}
\hline Module & Power consumption \\
\hline Solar cell & 0 \\
\hline Thresholding circuit & $0.5 \mu \mathrm{W}$ \\
\hline Scatterlight $(100 \mathrm{kHz}-1 \mathrm{MHz})$ & $19.5 \mu \mathrm{W}-120 \mu \mathrm{W}$ \\
\hline
\end{tabular}

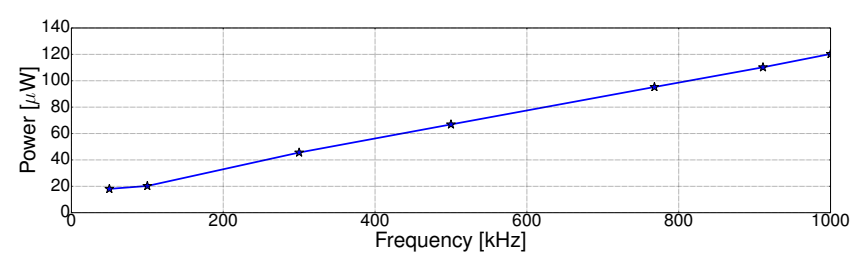

Figure 5: Power consumption of VLM at $2 \mathrm{~V}$. At a $100 \mathrm{kHz}$ frequency for transmission, the VLM consumes $20 \mu \mathrm{Ws}$.

As an end-device, we leverage a CC1310 transceiver. We can also leverage commodity wireless devices that surround us: Zhang et al. demonstrate that wireless devices equipped with WiFi and BLE radios can perform channel sensing and receive frequency shifted backscatter transmissions [16].

\section{PRELIMINARY RESULTS}

Experiment setup. We evaluate our system in two different environments which are representative of our target applications. We perform the gesture recognition and presence sensing experiment in our office. We also perform the presence sensing experiment outside the university building on a bright and sunny day. Indoors, the light levels were $100 \mathrm{~lx}$ which is much lower than typical indoor illumination. For the outdoor experiment, the light was very bright, approximately $80000 \mathrm{~lx}$. We note that light sensors commonly employed on VLS systems do not operate under such diverse and extreme light conditions due to the saturation effect [3]. As an energy harvester, we use the TI BQ25570 because it can operate at very low input voltages. We use a capacitor of size $22 \mu \mathrm{F}$, which is comparable to other energy harvesting platforms such as CRFIDs. Under indoor light, we use the thin film solar cell for harvesting. Outdoors, however, since it is bright we are able to use a much smaller solar cell (Ixys KXOB22, 22x7 mm) for harvesting.

At the VLM, we keep track of the analog signal from the solar cell, and the output of the thresholding circuit using a logic analyser. The analog signal serves as a ground truth for the experiment. We use a VERT900 antenna on the tag and the carrier generator.

Power consumption. To measure the power consumption, we first connect the VLM in series with a Fluke multimeter, then we vary the oscillator frequency. As the power consumption increases with voltage [16], we keep the voltage to the lowest level required to operate all the modules, which we found to be $2 \mathrm{~V}$.

Table 1 demonstrates the power breakdown of the VLM. The thresholding circuit together with the solar cell consumes $0.5 \mu \mathrm{W}$ of power to sense and digitise shadow events. In the presence of a shadow, the VLM enables the Scatterlight mechanism to communicate the shadow event, which increases the overall power consumption. Figure 5 shows that Scatterlight, when enabled, consumes power proportional to the backscatter frequency. The power consumption of Scatterlight varies between $19.5 \mu \mathrm{W}$ to $120 \mu \mathrm{W}$ when 


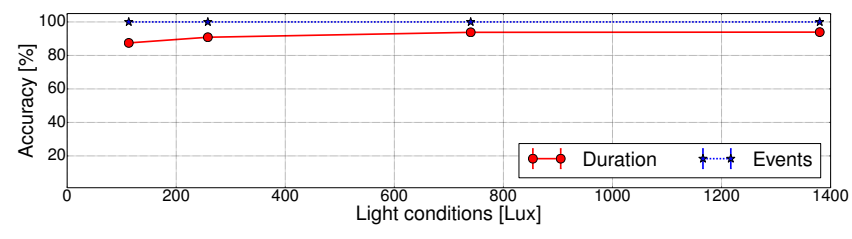

Figure 6: Accuracy of shadow sensing. Even in diverse light conditions, we detect all the shadow events. The accuracy to detect events in time decreases under low light.

backscattering at frequencies between $100 \mathrm{kHz}$ to $1 \mathrm{MHz}$. The energy harvested by the solar cell we employ is sufficient to power the VLM, even in darker light conditions (100 lx). Further, at a backscatter frequency of $100 \mathrm{kHz}$, the ultra-low-power nature of the VLM allows it to be operated on harvested energy from photodiodes [10]. Shadow sensing performance. First, we evaluate the performance of the VLM in terms of detecting shadow events under varying and representative light conditions for our target applications. We perform the experiment indoors, and cast a shadow on the solar cell a fixed number of times (60). We vary the light levels between $100 \mathrm{~lx}$ to $1400 \mathrm{~lx}$. We perform three instances of the experiment for each light condition. In the experiment, we use the number of shadow events successfully detected, and the accuracy in time of these successful events as performance metric. To find the accuracy in time, we compare the duration of the digitised sample with the corresponding analog sample from the ground truth data.

Figure 6 shows that VLM detects all the shadow events successfully even in low light conditions. However, the accuracy to detect events in time duration decreases with the light condition. In the future, we aim at improving this accuracy.

Communication range. Next, we evaluate the ability of the VLM to detect and communicate a shadow event in an indoor office and outdoors, for example, a shadow cast by a person near the VLM. We perform the experiment with the objective to determine the achievable range. For the indoor experiment, we place the tag and the receiver so that they are not in line-of-sight, as this represents the most challenging scenario for RF communication. We place the VLM on the ground and the carrier generator at a distance of $1 \mathrm{~m}$ from the tag. This distance between the carrier source and the tag is also used by state-of-the-art backscatter systems $[5,11,16]$.

We conduct the experiment by having a person walk and cast a shadow a fixed number of times (10) on the VLM's solar cell. We repeat each experiment three times. In the experiment, we were able to successfully detect all the shadow events. Further, Figure 7 shows that we are able to communicate to distances as high as $70 \mathrm{~m}$ indoors, even when the carrier generator and the receiver are separated by multiple walls. When we perform the experiment outdoors, we are able to communicate to distances as high as $330 \mathrm{~m}$. The achieved range is sufficient for our target applications.

Gesture detection. Finally, we investigate the ability of our system to detect simple hand gestures. In this preliminary work, we focus on three hand gestures: swipe, two taps and four taps, which we illustrate in Figure 9. We perform the experiment indoors, and locate the carrier generator source approximately $20 \mathrm{~m}$ from the VLM. The carrier generator is not in line-of-sight from the receiver.

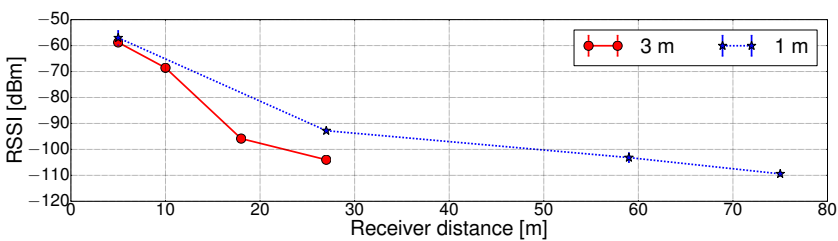

Figure 7: Communication range (indoors). We can communicate to distances of tens of meters. Carrier generator and tag were at separated by $1 \mathrm{~m}$ and $3 \mathrm{~m}$.

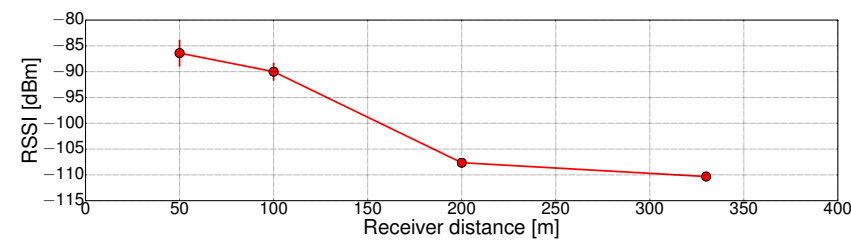

Figure 8: Communication range (outdoors). Even in extremely bright light conditions $(80000 \mathrm{~lx})$, we can sense presence, and communicate upto distances of $330 \mathrm{~m}$.
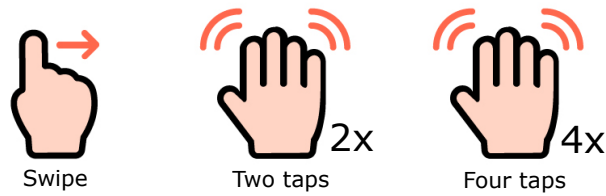

Figure 9: Supported hand gestures. We support three hand gestures: Swipe is represented by a single and brief hand movement, Two and Four taps are represented by fixed number of slower palm movements.

We place the VLM at a distance of $1 \mathrm{~m}$ from the receiver, a typical distance for transmissions from smart clothes to a wearable device.

To conduct the experiment, we perform hand gestures over the VLM's solar cell. We track the digital and analog signal from the VLM using a logic analyser, and also sample the RSSI at the RF receiver at an interval of $10 \mathrm{~ms}$ which is sufficient for detecting hand gestures occurring at the order of hundreds of milliseconds. Figure 10 demonstrates that each gesture causes unique patterns in the ambient light levels, as also observed by Kaholokula et al. [3]. However, unlike their design we use an energy efficient solar cell together with the thresholding circuit to digitise shadow events, and communicate them at $\mu \mathrm{Ws}$ using the Scatterlight mechanism.

Figure 10 shows that the unique patterns observed in ambient light levels (caused by hand gestures) are mirrored onto the backscattered signal and observed in the obtained RSSI samples through the CC1310 RF-receiver. We transfer these samples to a laptop which implements a simple binary classifier to identify these gestures. In our test conditions, we could correctly classify hand gestures in the RSSI samples with $100 \%$ accuracy. However, we expect it to be challenging to detect gestures with high accuracy under low ambient light conditions (e.g. below $100 \mathrm{~lx}$ ). In the future, we will explore other techniques to detect complex gestures even under varying light conditions. 


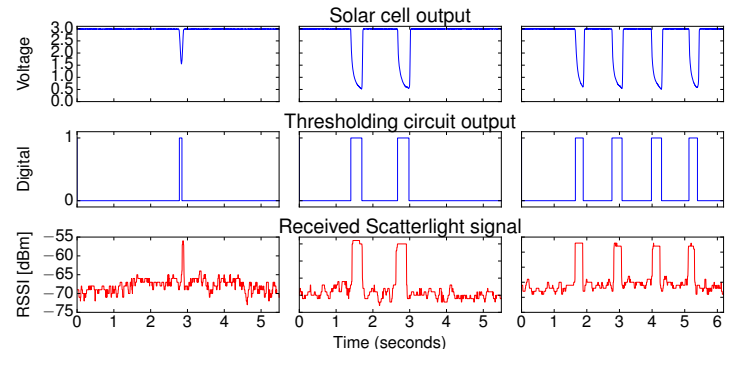

Figure 10: Sensing hand gestures. We detect three hand gestures (Swipe, Two taps, Four taps) at $20 \mu \mathrm{Ws}$ of power. The top two rows show the output at the VLM, the bottom row shows the received signal at the end-device.

\section{CONCLUSION AND FUTURE WORK}

We presented the first system that uses a solar cell to achieve sub $\mu \mathrm{Ws}$ of power to detect shadow events. Further, the system uses a novel mechanism to delegate sensor readings to the end-devices at $20 \mu \mathrm{Ws}$ of power. We demonstrated the ability to detect three hand gestures and sense the presence of people even in bright light conditions. We recognize the early design of our work, and in the future plan to address the following challenges:

Complex gestures. We presented preliminary results that show that it is possible to detect three hand gestures at a significantly lower power compared to existing systems. However, our initial results use a single VLM, even though existing systems demonstrate that it is possible to detect complex gestures using multiple light sensors [7, 8]. We will explore this possibility in our future work.

There is also a potential to improve the ability to detect hand gestures at the end-device (receiver). At present, we have assigned simple binary classifiers that operate on sampled RSSI readings for each of the three hand gestures. However, we can leverage more advanced machine learning methods to allow for classifying complex gestures. The key challenge is to keep the complexity of such algorithms within the constraints of low-end devices such as wearables with limited processing abilities.

Leveraging ambient wireless signals. A promising research direction is to utilise ambient wireless signals such as television [9] or FM signals [12] which would eliminate the need for a separate carrier generator device. Conceptually, our present design can backscatter ambient signals. The key challenge lies in discerning variations caused by gestures from inherent changes in the modulated RF signals at the end-device. One possible solution is to use averaging to separate slow varying shadow events at the end-device. We will explore this possibility in our future work.

Analog backscatter. In VLM, we digitse the analog output from the solar cell using a thresholding circuit. The thresholding circuit acts like a 1 bit ADC, and hence analog information about the intensity of the shadow or ambient light levels is lost in the digitisation process. To overcome this shortcoming, a promising research direction is to backscatter analog signals directly from the sensor without involving computational blocks as demonstrated by Talla et al. [10]. However, their design suffers from severe self-interference to weak backscattered signal and requires complex processing on SDRs to recover information.

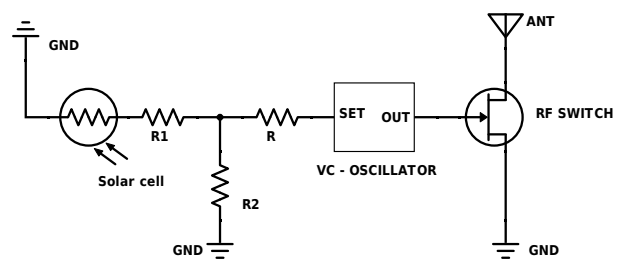

Figure 11: Analog backscatter. Transforming analog signal to corresponding change in the frequency of the backscatter signal enables transmitting of analog information at $\mu \mathrm{Ws}$.

We can overcome the high self-interference together with retaining analog information from the solar cell by improving Scatterlight using the analog backscatter design presented in Figure 11. The design instead of digitising the analog signal using a thresholding circuit, instead maps analog values to unique frequencies that are larger than $\Delta f$ required to reduce self-interference. We perform this by connecting the output of a solar cell to an ultra-low power voltage-controlled oscillator. The voltage-controlled oscillator generates a digital signal with a frequency corresponding to the analog input signal. The end-device reconstructs the analog signals by identifying the frequencies in the received backscattered transmissions. We leave a more detailed evaluation of analog backscatter based VLM to future work.

\section{ACKNOWLEDGEMENT}

We thank the anonymous reviewers for their insightful comments. This work has been funded by the Swedish Energy Agency (Energimyndigheten).

\section{REFERENCES}

[1] Mohamed Ibrahim et al. 2016. Visible Light Based Activity Sensing Using Ceiling Photosensors. In VLCS 2016.

[2] Vikram Iyer et al. 2016. Inter-technology backscatter: Towards internet connectivity for implanted devices. In ACM SIGCOMM 2016.

[3] M Kaholokula. 2016. Reusing Ambient Light to Recognize Hand Gestures. Dartmouth college. (2016).

[4] Bryce Kellogg, Vamsi Talla, and Shyamnath Gollakota. 2014. Bringing Gesture Recognition to All Devices. In USENIX NSDI 14.

[5] Bryce Kellogg, Vamsi Talla, Shyamnath Gollakota, and Joshua R Smith. 2016. Passive wi-fi: Bringing low power to wi-fi transmissions. In USENIX NSDI 2016.

[6] Ye-Sheng Kuo, Pat Pannuto, Ko-Jen Hsiao, and Prabal Dutta. 2014. Luxapose: Indoor Positioning with Mobile Phones and Visible Light. In ACM MOBICOM.

[7] Tianxing Li, Chuankai An, Zhao Tian, Andrew T Campbell, and Xia Zhou. 2015. Human sensing using visible light communication. In ACM MOBICOM 2015.

[8] Tianxing Li, Qiang Liu, and Xia Zhou. 2016. Practical human sensing in the light. In ACM MOBISYS 2016.

[9] Vincent Liu et al. 2013. Ambient Backscatter: Wireless Communication out of Thin Air. In ACM SIGCOMM 2013.

[10] Vamsi Talla, Bryce Kellogg, Shyamnath Gollakota, and Joshua R Smith. 2017. Battery-Free Cellphone. In ACM UBICOMP 2017.

[11] Ambuj Varshney et al. 2017. LoRea: A Backscatter Architecture that Achieves a Long Communication Range. In ACM SENSYS 2017.

[12] Anran Wang, Vikram Iyer, Vamsi Talla, Joshua R Smith, and Shyamnath Gollakota. 2017. FM Backscatter: Enabling Connected Cities and Smart Fabrics.. In NSDI.

[13] Zixiong Wang, Dobroslav Tsonev, Stefan Videv, and Harald Haas. 2015. On the design of a solar-panel receiver for optical wireless communications with simultaneous energy harvesting. IEEE 7SAC 2015 (2015).

[14] Chi Zhang and Xinyu Zhang. 2016. LiTell: Robust Indoor Localization Using Unmodified Light Fixtures. In ACM MOBICOM 2016.

[15] Pengyu Zhang et al. 2014. Ekhonet: High speed ultra low-power backscatter for next generation sensors. In ACM MOBICOM 2014.

[16] Pengyu Zhang et al. 2016. Enabling practical backscatter communication for on-body sensors. In ACM SIGCOMM 2016.

[17] Shilin Zhu et al. 2017. Enabling High-Precision Visible Light Localization in Today's Buildings. In ACM MOBISYS 2017. 